\title{
Assistenza e Tecnologia: possono interagire?
}

Per poter stabilire se la tecnologia condizioni in qualche modo l'assistenza infermieristica, è necessario innanzittutto fare chiarezza sul significato di "ASSISTENZA INFERMIERISTICA".

Secondo Virginia Henderson, riconosciuta esperta di modelli concettuali dell'assistenza, "La funzione specifica dell'infermiera è quella di assistere l'individuo sano o malato, per aiutarlo a compiere tutti quegli atti tendenti al mantenimento della salute o al raggiungimento della guarigione (o a prepararlo ad una morte serena), atti che compirebbe da solo se disponessse della forza, della volontà o delle condizioni necessarie. Inoltre è compito dell' infermiera quello di fa vorire la partecipazione attiva del paziente in modo da aiutarlo a conquistare il più rapidamente possibile la propria indipendenza". Dunque l'obiettivo primario dell'assistenza è l'uomo nella sua totalità.

L'assistenza infermieristica ottimale deve necessariamente basarsi su due elementi:

1) conoscenza delle tecniche fondate su principi scientifici;

2) capacità di stabilire una comunicazione empatica con l'individuo/paziente.

Tali elementi devono completarsi a vicenda: un'infermiera efficiente sotto il profilo tecnicostrumentale può non essere efficace se non svolge il suo ruolo di comunicazione/relazione con la necessaria sensibilità.

Indubbiamente i compiti tecnico-strumentali sono i più facili da apprendere perchè sono i più concreti e precisi, mentre le funzioni di comunicazione/relazione richiedono una notevole abilità e implicano un considerevole coinvolgimento personale.

Probabilmente questo è il motivo per cui molto spesso nella nostra realtà la tecnica ha il sopravvento sui rapporti interpersonali. Ma l'infermiera non può e non deve dimenticare che la sua relazione col paziente non è un rapporto casuale, ma ha il preciso scopo di facilitarne l'addattamento positivo dell'individuo allo stress cui è sottoposto. L'infermiera deve aiutare l'individuo ad accettare la propria malattia e a modificare se necessario la propria esistenza in rapporto alla mutata situazione.
Riuscire a porre sempre il paziente nella sua globalità al centro di ogni atto assistenziale è indubbiamente un compito che richiede molto impegno all'infermiera la quale deve come primo compito aiutare l'individuo a identificare e a esprimere i propri bisogni. Per esprimere tali bisogni il paziente può ovviamente a vvalersi della comunicazione verbale; a volte ciò non è possibile per cui diventa determinante la capacità di saper osservare attentamente qualsiasi espressione o comportamento del malato. Inoltre non bisogna dimenticare che allo stress della malattia l'individuo reagisce con dei meccanismi di difesa ed è qui che l'infermiera gioca un ruolo decisivo riuscendo a dare un significato a tali risposte, potenziando le forze positive del malato affinchè trovi in se stesso la giusta capacità di reazione per conservare la propria integrità.

L'infermiera è colei che ha contatti più frequenti col malato, nella giornata e nel tempo ed inoltre è l'operatore sanitario più accessibile e più disponibile. Questi aspetti rivelano il carattere unico dell'infermiera come membro dell'équipe sanitaria, da qui la necessità di definire attivamente le proprie funzioni contestando se occorre la pratica corrente, le strutture esistenti e i rapporti di potere. La necessità di rompere con la pratica corrente e con le strutture esistentioggi è più che mai sentita. Non si può negare di vivere un momento di disagio e di insoddisfazione riconducibile probabilmente al modo di "curare" prevalentemente tecnico.

Le prestazioni che la struttura richiede all'infermiera sono una serie di compiti codificati che tengono in scarsa considerazione la complessità dei bisogni della persona e della reale efficacia degli interventi erogati. Ci si muove all'insegna di uno slogan indegno per una società che voglia definirsi civile: "TUTTO E TUTTI UGUALE PER TUTTI"

Qualunque sia il settore in cuil'infermiera opera, le difficoltà che deve affrontare per riuscire a erogare un'assistenza che tenga conto del malato in quanto uomo sono molte: inadeguata formazione scolastica, organici insufficienti, esecuzione di una serie di atti medici delegati che la costringono a tralasciare alcune delle sue specifiche man- 
sioni.

L'infermiera di dialisi oltre ad affrontare le suddette difficoltà deve anche fare i conti con l'utilizzo delle apparecchiature tecnologiche che condizionano pesantemente la specificità dell'assistenza: il rischio è che le attenzioni dell'infermiera non abbiano come obiettivo principale il paziente ma l'apparecchiatura.

L'infermiera di dialisi impiega molte delle sue energie per la preparazione e la manutenzione dell'apparecchio, energie che vengono sottratte all'assistenza diretta.

L'uso di un'apparecchiatura può portare l'infermiera ad essere più attenta ai segnali inviati dalla macchina più che ai sintomi o ai bisogni espressi dal paziente. Durante la seduta stessa, la maggior parte degli interventi infermieristici sono indirizzati ai monitor più che all'individuo.

Ora è indubbio che il benessere del paziente è consequenziale al buon funzionamento dell'apparecchio, ma tutto ciò risponde solo a una parte dell'assistenza, cioè la cura della malattia, mentre lo specifico professionale infermieristico impone di curare l'uomo e non solo la malattia.

Fermo restando la necessità che a occuparsi dell'assistenza al dializzato sia una sola figura professionale, cioè l'infermiera è necessario che le apparecchiature utilizzate siano affidabili ed estremamente semplici, affinchè l'infermiera sia più libera di concentrare la propria attenzione sul paziente.

E inoltre necessario che nei centri dialisi sia sempre presente un tecnico specializzato capace di occuparsi della manutenzione ordinaria e straordinaria delle apparecchiature. In tal modo è possibile garantire la massima efficienza dei reni artificiali e l'infermiera potrà così esprimere la propria professionalità offrendo al paziente un'assistenza globale efficiente sì, ma soprattutto efficace.

\section{M.T. Parisotto}

